\title{
Urinary gonadotropin peptide as acute phase reactant: transient elevation after operation for digestive diseases
}

\author{
Yoshiharu Motoo, Hiroyuki Watanabe, Takashi Okai and Norio Sawabu \\ Department of Internal Medicine and Medical Oncology, Cancer Research Institute, Kanazawa University, Kanazawa, Japan \\ (Correspondence should be addressed to Y Motoo, Department of Internal Medicine and Medical Oncology, Cancer Research Institute, \\ Kanazawa University, 4-86 Yoneizumi, Kanazawa 921-8044, Japan)
}

\begin{abstract}
Objective: In order to characterize urinary gonadotropin peptide (UGP) as an acute phase reactant, we focused on the UGP levels after surgical operation.

Design: Fifty cases of gastrointestinal cancer, 4 cases of cancers of other organs and 13 cases of benign digestive diseases were enrolled into this study.

Methods: UGP levels were measured using an enzyme immunoassay before and after surgery.

Results: Fifty-four $(80.6 \%)$ of the 67 cases studied showed transient elevations of UGP. Both urinary interleukin (IL)-6 and LH levels were also increased transiently in 49 cases (73.1\%). All of these three factors were increased in 38 cases $(56.7 \%)$, and in $32(84.2 \%)$ of these 38 cases, the order of peak appearance was as follows: IL-6, LH, and UGP. The UGP levels in the group of total gastrectomy were significantly higher than those in the group of partial gastrectomy.

Conclusions: These results suggest that UGP shows a transient peak after surgery, correlating with levels of cytokines such as IL-6. UGP may be an acute phase reactant, and its levels are correlated with the grade of surgical stress.
\end{abstract}

European Journal of Endocrinology 140 555-560

\section{Introduction}

Urinary gonadotropin peptide (UGP), a $10.5 \mathrm{kDa}$ peptide, is a metabolite of human chorionic gonadotropin (hCG) $\beta$ subunit, and has an epitope which appears only when hCG $\beta$ is degraded and excreted into the urine (1).

UGP is used as a tumor marker for gynecologic (2-7) and urologic $(8-10)$ malignancies. We have reported the clinical significance of UGP as a tumor marker for gastrointestinal cancers, showing a positive rate of approximately $60 \%$ in pancreatobiliary cancers and a low false-positive rate in benign digestive diseases (11). UGP is reported to be a tumor marker for lung cancer (12) and other cancers (13).

During our study on the measurement of UGP in cancers, we noticed that several patients with benign diseases such as gastric leiomyoma showed a transient elevation of UGP after surgery (Y Motoo, unpublished data). Recently, Funakoshi et al. (14) reported that UGP levels transiently increased after the onset of acute pancreatitis. UGP is also reported to be useful for the screening of Down's syndrome (15).

In the present study, we investigated UGP levels before and after surgery in 67 patients.

\section{Subjects and methods}

\section{Patients}

Fifty patients with cancers of the digestive organs (stomach 36, colon and rectum 8 , liver 3 , pancreas 2 , esophagus 1), 4 patients with cancers of nondigestive organs (thyroid gland 3 and mammary gland 1 ) and 13 patients with benign digestive diseases (gallbladder stone 8, gallbladder adenomyomatosis, gallbladder polyp, gastric adenoma, gastric leiomyoma, abdominal wall hernia, one case for each disease) were included in this study. The total number of the cases of digestive diseases was 63 (50 cancers and 13 benign diseases). The diagnosis was confirmed by pathologic examinations after operation in all the cases studied. There were 38 males and 29 females (18 menopausal), and the ages ranged from 36 to 76 (mean 65.8) years.

\section{Urine collection}

First-morning or random urine collection was performed. A 24-h urine collection was not done. Fresh urine $(10 \mathrm{ml})$ was stored at $-20^{\circ} \mathrm{C}$ until assay without 
any preservatives. There was no pretreatment such as $\mathrm{pH}$ adjustment.

\section{UGP assay}

A sandwich enzyme immunoassay kit (ELNAS UGP: TFB Inc., Tokyo, Japan) was used. This ELISA kit utilizes the monoclonal antibody B210 which reacts only with the core fragment of urinary hCG $\beta$ (16). Crossreactivity of UGP to the following hormones was less than $0.25 \%$ (Y Shirakawa, personal communication): luteinizing hormone (LH), LH $\beta$, hCG and hCG $\beta$. The minimum detectable concentration of the assay was $0.1 \mathrm{fmol} / \mathrm{ml}$. Using a tentative cut-off of $5 \mathrm{fmol} / \mathrm{mg}$ creatinine $(\mathrm{Cr})$, UGP was positive in $8.8 \%$ of healthy women (premenopausal 5.2\%, postmenopausal 11.6\%). UGP was positive in $7.2 \%$ of women with benign diseases, whereas it was positive in $52 \%$ of ovarian cancer patients (2). The interassay variation was approximately $10 \%(2)$. Urinary $\mathrm{Cr}$ levels were determined to normalize UGP values to account for urine concentration variability. Urinary $\mathrm{Cr}$ was determined by the alkaline picrate method, and UGP values were expressed as $\mathrm{fmol} / \mathrm{mg} \mathrm{Cr}$ (17). Cut-off values were defined as the mean \pm 2 s.D. in healthy controls $(1.7 \mathrm{fmol} / \mathrm{mg} \mathrm{Cr}$ in males and $2.5 \mathrm{fmol} /$ $\mathrm{mg} \mathrm{Cr}$ in females) at first. However, according to previous reports $(8,18)$ on a large number of healthy volunteers and various diseases, suitable cut-off values were considered to be $2.2 \mathrm{fmol} / \mathrm{mg} \mathrm{Cr}$ for males and $3.8 \mathrm{fmol} / \mathrm{mg} \mathrm{Cr}$ for females (11). Standard UGP was included in the kit. This standard was a purified hCG $\beta$ subunit from the National Institutes of Health.

\section{Urinary interleukin (IL)-6 and LH assay}

Urinary IL-6 was determined with an enzyme immunoassay kit (Toray Industry, Inc., Tokyo, Japan), and urinary LH was measured with an immunoradiometric assay kit (SPAC-S LH kit; Daiichi Radioisotope Labs, Ltd,
Tokyo, Japan). The cut-off value for IL- 6 was $4 \mathrm{pg} / \mathrm{mg}$ $\mathrm{Cr}$. The cut-off value for $\mathrm{LH}$ was $17 \mathrm{mIU} / \mathrm{mg} \mathrm{Cr}$ for males and $30 \mathrm{mIU} / \mathrm{mg} \mathrm{Cr}$ for females.

\section{Statistical analysis}

Student's t-test with Welch's correction was used for the comparison of the mean values of two groups. Differences between groups were considered significant if the $P$ value was less than 0.05 .

\section{Results}

The changes in UGP, urinary LH (u-LH) and urinary IL-6 (u-IL-6) were classified as follows. (A) Preoperatively low with a postoperative transient elevation; (B) preoperatively low and continuously high postoperatively; (C) preoperatively high and further transient elevation postoperatively; (D) preoperatively high and decreased postoperatively; (E) preoperatively high and further elevation postoperatively; (X) miscellaneous.

Table 1 shows the results of the above classification. Pattern A was seen in $74.6 \%$ in UGP, $73.1 \%$ in u-LH, and $67.2 \%$ in u-IL-6. C-reactive protein (CRP) was transiently elevated in $86.9 \%$ of the cases studied (data not shown). The percentage of males and females exhibiting pattern A was $76.3 \%$ and $72.4 \%$ in UGP, $81.6 \%$ and $62.1 \%$ in $\mathrm{u}-\mathrm{LH}$, and $71.1 \%$ and $62.1 \%$ in u-IL-6. All three factors tended to be increased in males, and a significant difference was recognized in $\mathrm{u}-\mathrm{LH}$ $(P<0.02)$. There was no significant difference in the percentage with pattern A in UGP between males and females.

As shown in Table 2, the preoperative levels of UGP were elevated in 5 cases and below the cut-off values in 62 cases. Fifty $(80.6 \%)$ of these 62 cases showed transient elevations of UGP after operation. Four $(80 \%)$ of the 5 preoperatively high cases also showed a transient increase in UGP values during postoperative

Table 1 The patterns of changes of UGP, u-LH and U-IL-6 before and after operation.

\begin{tabular}{|c|c|c|c|c|c|c|c|}
\hline & A & B & C & D & $E$ & $x$ & Total \\
\hline \multicolumn{8}{|l|}{ UGP } \\
\hline Male & 29 & 3 & 1 & 0 & 0 & 5 & 38 \\
\hline Female & 21 & 1 & 3 & 0 & 0 & 4 & 29 \\
\hline \multicolumn{8}{|l|}{$\mathrm{u}-\mathrm{LH}$} \\
\hline Male & 31 & 1 & 0 & 1 & 0 & 5 & 38 \\
\hline Female & 18 & 3 & 0 & 1 & 1 & 6 & 29 \\
\hline Premenopausal & 7 & 1 & 0 & 0 & 0 & 3 & 11 \\
\hline Postmenopausal & 11 & 2 & 0 & 1 & 1 & 3 & 18 \\
\hline \multicolumn{8}{|l|}{ u-IL-6 } \\
\hline Male & 27 & 2 & 3 & 1 & 0 & 5 & 38 \\
\hline Female & 18 & 3 & 1 & 2 & 2 & 3 & 29 \\
\hline
\end{tabular}

(A) Preoperatively low, postoperatively high transiently; (B) preoperatively low, postoperatively high; (C) preoperatively high, postoperatively higher transiently; (D) preoperatively high, postoperatively low; (E) preoperatively high, postoperatively higher; $(X)$ unclassified. 
Table 2 Comparison of the levels of UGP before and after operation.

\begin{tabular}{lccc}
\hline & \multicolumn{2}{c}{ UGP $(\mathrm{fmol} / \mathrm{mg} \mathrm{Cr})$} & \\
\cline { 2 - 3 } & Before & After (peak) & P value \\
\hline $\begin{array}{l}\text { Malignant diseases } \\
(n=54)\end{array}$ & $1.39 \pm 3.55$ & $7.37 \pm 9.41$ & 0.00003 \\
$\begin{array}{l}\text { Low preoperatively } \\
(n=49)\end{array}$ & $0.49 \pm 0.48$ & $6.47 \pm 8.90$ & 0.0001 \\
$\begin{array}{l}\text { High preoperatively } \\
(n=5)\end{array}$ & $7.32 \pm 7.87$ & $16.16 \pm 10.82$ & 0.178 \\
$\begin{array}{l}\text { Benign diseases } \\
(n=13)\end{array}$ & $0.88 \pm 1.12$ & $1.68 \pm 1.91$ & 0.333 \\
$\begin{array}{l}\text { Total } \\
(n=67)\end{array}$ & $1.40 \pm 3.37$ & $6.73 \pm 9.08$ & 0.00003 \\
\hline
\end{tabular}

follow-up. Therefore, 54 (80.6\%) of the 67 cases studied showed transient elevations of UGP. Table 2 shows the preoperative and postoperative peak values of UGP. The postoperative peak values were significantly higher than those before operation especially in malignant diseases.

Urinary LH levels were increased preoperatively in 7 cases ( 3 males and 4 postmenopausal women) and below the cut-off value in 60 cases. A transient elevation of $\mathrm{u}-\mathrm{LH}$ was seen in $49(73.1 \%, 31$ males, 7 premenopausal and 11 postmenopausal women) of the 67 cases.

Urinary IL- 6 was increased in 12 cases and below the cut-off value in 55 cases preoperatively. Forty-five $(67.2 \%)$ of the 67 cases showed a transient elevation of u-IL-6 levels postoperatively. In addition, CRP was transiently elevated in $56(83.6 \%)$ of the 67 cases studied.

Of the 67 patients, all of these three factors were increased in 38 cases $(56.7 \%)$, and $32(84.2 \%)$ of these 38 cases showed a peak of the three factors in the order of u-IL-6, u-LH and UGP (or at the same time). There was no significant correlation among the peaks of the three factors, or between the peak of each factor and that of CRP. As for CRP, the peak appeared at almost the same time as that of u-IL-6, prior to the appearance of UGP. As far as we could tell, there was no influence of renal dysfunction. There could be some non-specific effects of surgery or drugs on the UGP assay, but no specific drugs were used on the patients who showed UGP elevation after operation. We measured UGP and serum hCG in 18 patients before operation, but serum hCG was measured in only 5 patients after operation. There was no transient elevation of serum hCG in patients who showed a transient elevation of UGP.

\section{Case presentation}

Case 1 (Fig. 1). A 55-year-old postmenopausal female who underwent total gastrectomy due to schirrhous carcinoma. After operation, high fever was prolonged for 3 weeks, and paralytic ileus developed. During the postoperative period, u-IL-6, u-LH and UGP levels rose to the same peak on day 14 , decreased once, re-elevated, and were prolonged for 3 weeks.

Case 2 (Fig. 2). A 69-year-old male who underwent subtotal gastrectomy due to advanced gastric cancer. Preoperative levels of u-IL-6, u-LH and UGP were low, but postoperative levels of $\mathrm{u}-\mathrm{IL}-6$ peaked on day 7, while those of $\mathrm{u}-\mathrm{LH}$ and UGP peaked on day 14. The levels of

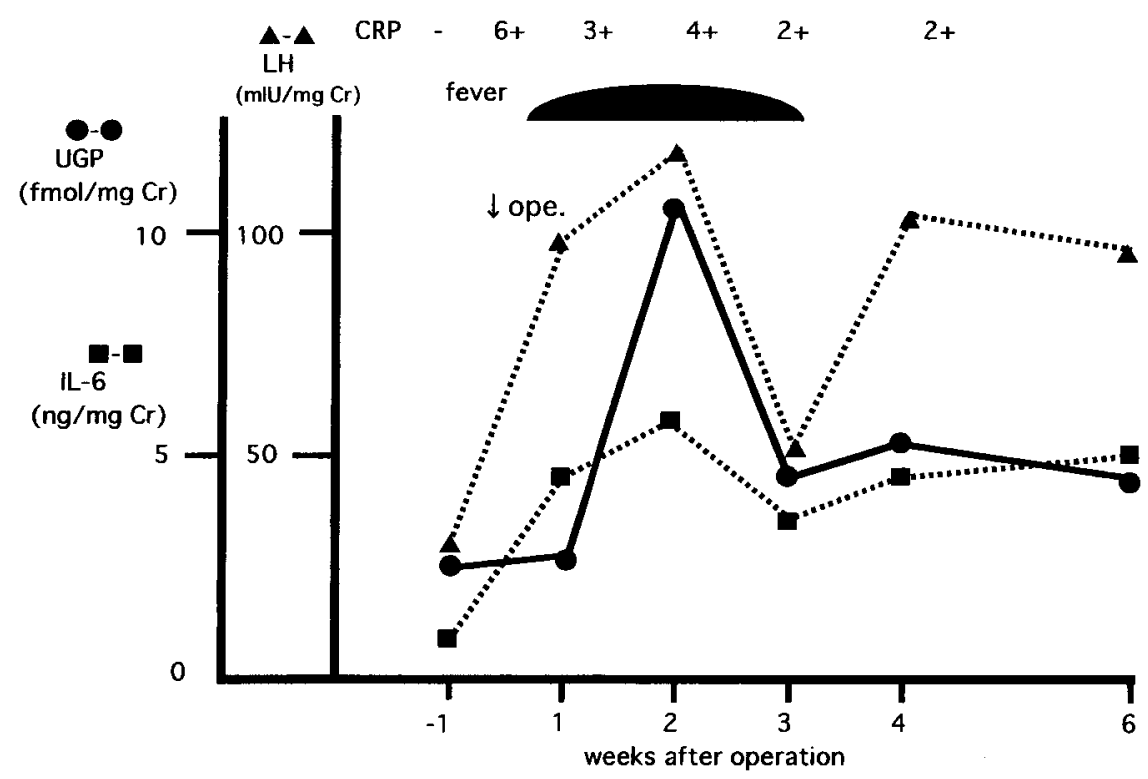

Figure 1 Case 1. A 55-year-old female who underwent total gastrectomy due to advanced gastric cancer. ope. $=$ operation. 


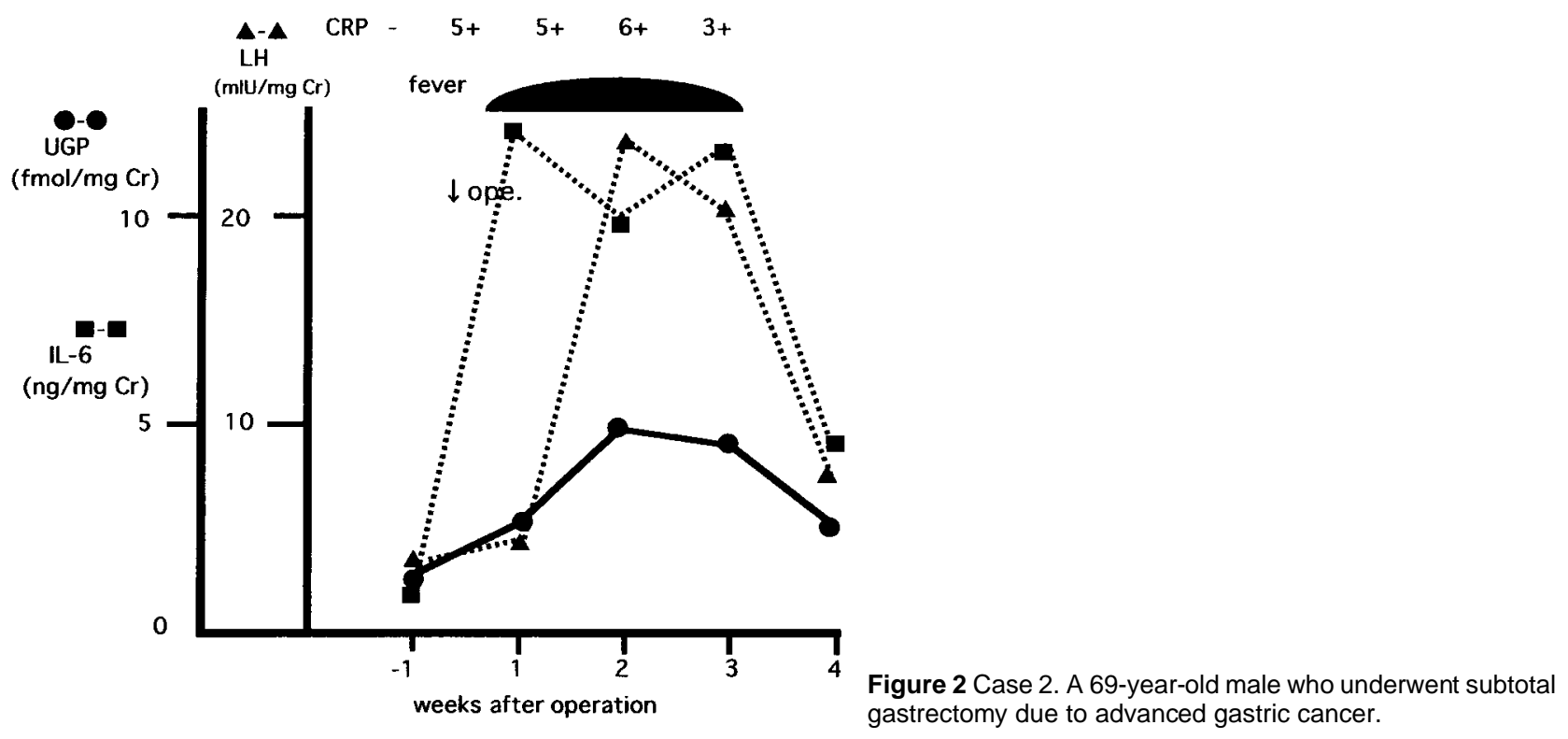

these three factors remained elevated, and there were several complications such as bacterial infection.

Case 3 (Fig. 3). A 42-year-old premenopausal female who underwent distal partial gastrectomy due to early gastric cancer. Preoperative levels of u-IL-6, u-LH and UGP were low, but postoperative levels of u-IL-6 and $\mathrm{u}-\mathrm{LH}$ peaked on day 3, and UGP levels peaked on day 7 . All levels normalized on day 14 , and she was discharged without any postoperative complications.

The relationship between the peak levels of UGP and the grade of surgical stress is shown in Fig. 4. The UGP

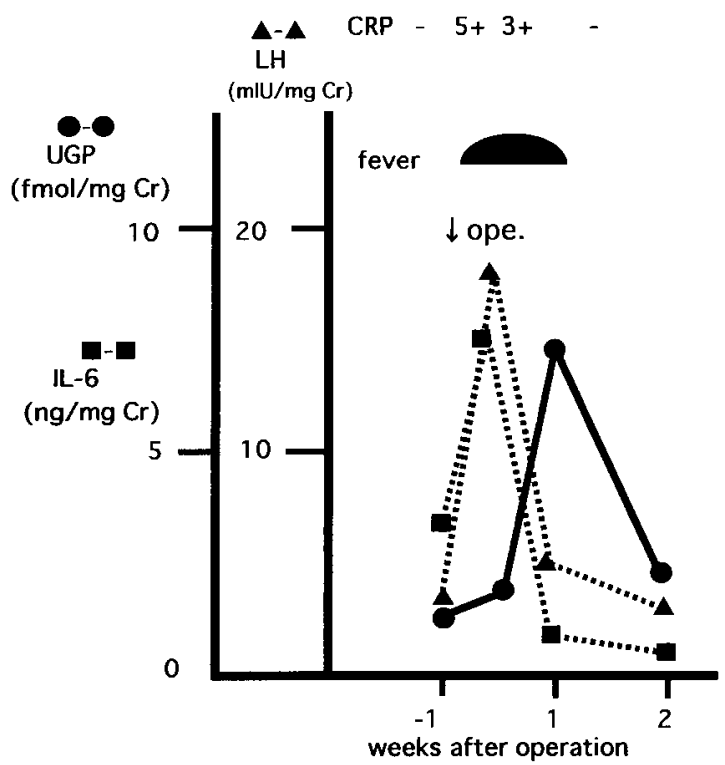

Figure 3 Case 3. A 42-year-old female who underwent distal partial gastrectomy due to early gastric cancer. peaks tended to elevate as the surgical stress was increased from laparoscopic surgery to total gastrectomy. The UGP peak in the total gastrectomy group was significantly higher than that in the partial gastrectomy group $(P<0.05)$. Although the $\mathrm{u}$-LH peak in the subtotal gastrectomy group was significantly higher than that in the partial gastrectomy group $(P<0.05)$, there was no significant difference in u-IL-6 levels among these groups.

\section{Discussion}

It is known that levels of acute phase reactants such as CRP and various cytokines are elevated after surgical stress (19). In this study, we have shown for the first time that UGP is elevated transiently after surgery. Recently, Funakoshi et al. (14) reported a transient elevation of UGP in patients with acute pancreatitis. Iles (15) reported the elevation of UGP in Down's syndrome. Other than these reports, there has been no publication on a UGP elevation unrelated to cancer.

UGP is derived from the ectopically produced hCG $\beta$ in non-chorionic tumor cells (20), and is used as a tumor marker (2-13). However, there have been some reports suggesting that the UGP elevation is derived from stromal cells such as fibroblasts in regenerating tissues. For instance, a transient elevation of UGP in acute pancreatitis as mentioned above (14), the presence of detectable UGP in healthy males and male patients with benign diseases (18), and a transient elevation of UGP after operation for benign digestive diseases (Y Motoo, unpublished data).

The detection of UGP in healthy males is considered to be due to the small amount of hCG $\beta$ secreted in tissues with a rapid cell cycle such as normal gastrointestinal 


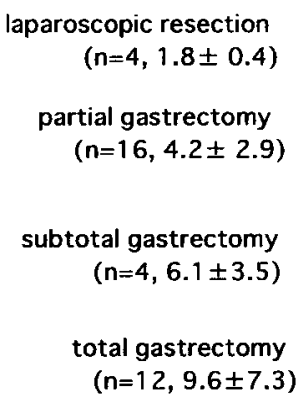

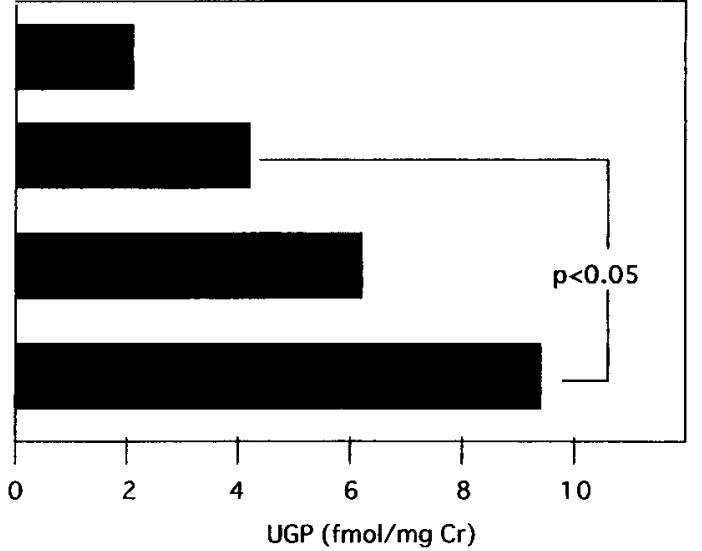

Figure 4 The peak levels of UGP in patients with different surgical stress. mucosa (20). The UGP ELISA kit used in this study has a detection limit of $0.1 \mathrm{fmol} / \mathrm{ml}$, approximately 20-fold more sensitive than an hCG $\beta$ assay $(2.3 \mathrm{fmol} / \mathrm{ml})$. Such sensitivity made it possible to detect the baseline levels of UGP in males and serial changes in UGP before and after surgery.

In inflammatory reactions, IL-6 is produced in lymphocytes, macrophages, fibroblasts, and vascular endothels. IL- 6 acts on hypothalamus and stimulates the secretion of corticotropin-releasing hormone (CRH). CRH stimulates pituitary adrenocorticotropic hormone (ACTH) and the level of adrenal glucocorticoid (GC) is elevated. In addition, after ACTH secretion, LH, which is suppressed by GC, is secreted as a result of a rebound phenomenon (or supplementary action of anterior pituitary gland) or direct pituitary stimulation by IL-6. Furthermore, as stated above, by a mechanism different from the cytokine-pituitary hormone interaction (21), $\mathrm{hCG} \beta$ is produced and secreted in the regenerating tissues at inflammatory sites. Yet another possible source of hCG $\beta$ core fragment as well as human (h) LH core fragment that has been reported in the literature (22) may be the pituitary gland.

As for clinical interest, UGP's most interesting characteristic is that it reflects clinical healing of injury (surgical insult). Proinflammatory cytokines are useful for the early detection of inflammation, but they are sometimes increased non-specifically. The elevation of UGP suggests the presence of not only inflammation but also regenerative process in response to it. The normalization of UGP might mean the completion of the regenerative process after surgical stress. However, tissue sampling of the regenerative area after surgery is almost impossible especially during the acute phase. Therefore there has been no evidence of $\mathrm{hCG} \beta$ production in regenerative tissues.

We measured the levels of $\mathrm{u}-\mathrm{IL}-6$ as a representative inflammatory cytokine. The measurement of $\mathrm{u}$-LH was carried out because of the structural similarity between hCG and LH (23-25). Concomitant elevation of u-LH and UGP could imply that UGP may be a derivative of
$\mathrm{hLH} \beta$ rather than of hCG $\beta$. Iles et al. (25) reported that the predominant $\beta$ core-like material present in the urine of postmenopausal woman may be a derivative of $\mathrm{hLH} \beta$ rather than of hCG $\beta$. UGP levels are also influenced by the menstrual cycle (26). We had to ensure that the changes of the two hormones differed at surgical stress although the two assay systems had no cross-reactivity. If the timing of the peak appearance of these hormones was the same, we considered that at least the peak was not delayed, and studied the order of the peak of each hormone.

As a result, u-IL-6, u-LH and UGP appeared in this order at surgical stress. That is, the reaction began with the production of IL- 6 and progressed to the secretion of pituitary gonadotropin (LH) and finally the production of non-pituitary gonadotropin (UGP). Interestingly, the patients with prolonged elevation of UGP showed poor recovery. There was a significant correlation between the UGP peak and surgical stress. From these results, it is suggested that UGP has the characteristics of an acute phase reactant and that the UGP peak can provide valuable information on the postoperative condition of patients.

In this study we confirmed the transient elevation of UGP after surgery in 54 patients. Together with a report on the transient elevation of UGP in acute pancreatitis (14), our findings suggest that a similar phenomenon occurs in other conditions which have acute inflammatory changes. The transient postoperative elevation of UGP suggests that UGP is an acute phase reactant. The local production of hCG $\beta$ in regenerative or recovering tissues needs to be confirmed.

In conclusion, we report for the first time that UGP acts as an acute phase reactant. UGP is elevated transiently after surgery without relation to malignancy, gender, organ or tissue.

\section{References}

1 Birken S, Armstrong EG, Gawinowicz Kolks MA, Cole LA, Agosto GM, Krichevsky A et al. Structure of the human chorionic 
gonadotropin $\beta$-subunit fragment from pregnant urine. Endocrinology $1988123572-583$.

2 Cole LA, Wang Y, Elliott M, Latif M, Chambers JT, Chambers SK et al. Urinary human chorionic gonadotropin free $\beta$-subunit and $\beta$-core fragment: A new marker of gynecological cancers. Cancer Research 198848 1356-1360.

3 Neven P, Iles RK, Lee CL, Hudson CN, Shepherd JH \& Chard T. Urinary chorionic gonadotropin subunits and beta-core in nonpregnant women. A study of benign and malignant gynecologic disorders. Cancer 199371 4124-4130.

4 Carter PG, Iles RK, Neven P, Ind TE, Shepherd JH \& Chard T. The prognostic significance of urinary beta core fragment in premenopausal women with carcinoma of the cervix. Gynecologic Oncology $199455271-276$.

5 Carter PG, Iles RK, Neven P, Ind TE, Shepherd JH \& Chard T. Measurement of urinary beta core fragment of human chorionic gonadotrophin in women with vulvovaginal malignancy and its prognostic significance. British Journal of Cancer 199571 350353.

6 Cole LA, Tanaka A, Kim GS, Park SY, Koh MW, Schwartz PE et al. Beta-core fragment (beta-core/UGF/UGP), a tumor marker: a 7-year report. Gynecologic Oncology 199660 264-270.

7 Schwarz-Roeger U, Petzoldt B, Waldschmidt R, Walker RP, Bauknecht T \& Kiechle M. UGP - a tumor marker of gynecologic and breast malignancies? Specificity and sensitivity in pretherapeutic patients and the influence of hormonal substitution on the expression of UGP. Anticancer Research 199717 3041-3045.

8 Yamanaka N, Kawabata G, Morisue K, Hazama M \& Nishimura R. Urinary hCG $\beta$-core fragment as a tumor marker for bladder cancer. Japanese Journal of Urology 199384 700-706.

9 Iles RK, Lee CL, Oliver RT \& Chard T. Composition of intact hormone and free subunits in the human chorionic gonadotrophinlike material found in serum and urine of patients with carcinoma of the bladder. Clinical Endocrinology 199033 355-364.

10 Iles RK, Persad R, Trivedi M, Sharma KB, Dickinson A, Smith P et al. Urinary concentration of human chorionic gonadotrophin and its fragments as a prognostic marker in bladder cancer British Journal of Urology 199677 61-69.

11 Motoo Y, Watanabe H, Yamaguchi Y, Mouri I, Fujii T, Yamakawa $\mathrm{O}$ et al. Urinary gonadotropin peptide in patients with cancers of digestive organs. Anticancer Research 199616 2041-2048.

12 Yoshimura M, Nishimura R, Murotani A, Miyamoto Y, Nakagawa $\mathrm{T}$, Hasegawa $\mathrm{K}$ et al. Assessment of urinary beta-core fragment of human chorionic gonadotropin as a new marker of lung cancer. Cancer $1994732745-2752$.

13 von Keist S, Walker B \& Walker R. Assessment of urinary gonadotropin in solid carcinomas other than gynecological tumors. Journal of Clinical Laboratory Analysis $199610184-192$.

14 Funakoshi A, Wakasugi H \& Shinozaki H. Two case studies of acute pancreatitis with transient elevation of urinary gonadotropin peptide (UGP) after IL-6 elevation. Journal of the Japan
Pancreas Society 199611 449-454 (In Japanese with English Abstract).

15 Iles RK. Urinary analysis for Down's syndrome: is the measurement of urinary beta-core the future of biochemical screening for Down's sundrome? Early Human Development 199647 S41-S45.

16 Krichevsky A, Birken S, O'Connor J, Bikel K, Schlatterer J, Yi C et al. Development and characterization of a new, highly specific antibody to the human chorionic gonadotropin- $\beta$ fragment. Endocrinology 1991128 1255-1264.

17 Plebani M, Navaglia F, Bacelle L, Polonio S, Lazzari F \& Marchetti M. Urinary gonadotropin peptide: collection of specimens and cutoff levels. Journal of Clinical Laboratory Analysis $19959230-$ 233.

18 Hirota F. Clinical significance of UGP (urinary gonadotropin peptide) as a tumor marker for non-chorionic cancers. Journal of the Japan Society for Cancer Therapy 199631 21-36 (In Japanese with English Abstract).

19 Murata A, Ogawa M, Yasuda T, Nishijima J, Oka Y, Ohmachi Y et al. Serum interleukin 6, C-reactive protein and pancreatic secretory trypsin inhibitor (PSTI) as acute phase reactants after major thoraco-abdominal surgery. Immunological Investigations $199019271-278$.

20 Manabe T, Adachi M \& Hirao K. Human chorionic gonadotropin in normal, inflammatory, and carcinomatous gastric tissue. Gastroenterology 198589 1319-1325.

21 Imura H, Fukata J \& Mori T. Cytokines and endocrine function: an interaction between the immune and neuroendocrine systems. Clinical Endocrinology 199135 107-115.

22 Patton PE, Hess DL, Cook DM, Loriaux DL \& Braunstein GD. Human chorionic gonadotropin production by the pituitary gland in a premenopausal woman. American Journal of Obstetrics and Gynecology 1998178 1138-1142.

23 Lapthorn AJ, Harris DC, Littlejohn A, Lustbader JW, Canfield RE, Machin KJ et al. Crystal structure of human chorionic gonadotropin. Nature $1994369455-461$.

24 Birken S, Kovalevskaya G \& O'Connor J. Metabolism of hCG and hLH to multiple urinary forms. Molecular and Cellular Endocrinology $1996125121-131$.

25 Iles RK, Lee CL, Howes I, Davies S, Edwards R \& Chard T. Immunoreactive $\beta$-core-like material in normal postmenopausal urine: human chorionic gonadotrophin or LH origin? Evidence for the existence of LH core. Journal of Endocrinology $1992133459-$ 466.

26 Neven P, Iles RK, Howes I, Sharma K, Shepherd JH, Edwards R et al. Substantial urinary concentrations of material resembling beta-core fragment of chorionic gonadotropin beta-subunit in mid-menstrual cycle. Clinical Chemistry 199339 1857-1860.

Received 25 November 1998

Accepted 25 February 1999 